\title{
Danza-escritura y dramaturgia. Experiencias de un taller
}

\section{Dance-writing and dramaturgy. Experiences of a workshop}

\author{
Laura Ruiz Mondragón \\ Universidad de Lille 3, Francia. \\ Doctorado en curso: Artes: Estética, teoría y prácticas \\ Laboratorio: CEAC (Centre d'Étude des Arts Contemporains). \\ lauraruizdanza@gmail.com \\ hitps:// orcid.org/0000-000 1-5225-4780
}

\section{Resumen}

El presente texto forma parte del trabajo doctoral "Investigación-acción: la danzaescritura como posibilidad de auto-enunciación". Para la parte práctica de la investigación se impartió el Taller de danza y escritura a manera de laboratorio. Lo que se presenta en este artículo es una parte del análisis de lo acontecido durante el taller. Se profundiza sobre el funcionamiento de la dramaturgia en el diseño de las sesiones, en la organización de los textos y danzas individuales y colectivos que se produjeron durante el laboratorio, así como en el ensamblaje de los materiales para su presentación ante un público. La dramaturgia es la herramienta articuladora para estudiar el proceso creativo en el que cada participante encontró un espacio para su propia enunciación y las resonancias que tuvo ésta en el colectivo. Para abordar el análisis, se toman como punto de partida los planteamientos de André Lepecki sobre dramaturgia y los de Laurence Louppe sobre partitura.

Palabras clave: Danza, Escritura creativa, Dramaturgia.

\section{Abstract}

This text is part of the doctoral work "Research-action: dance-writing as a possibility of self-enunciation". For the practical part of the research, the Dance and writing workshop was given as a laboratory. What is presented in this article 
is a part of the analysis of what happened during the workshop. It delves into the operation of dramaturgy in the design of the sessions, in the organization of the individual and collective texts and dances that occurred during the laboratory, as well as in the assembly of the materials for presentation to an audience. Dramaturgy is the articulating tool to study the creative process in which each participant found a space for their own enunciation and the resonances that this had in the group. To address the analysis, the approaches of André Lepecki on dramaturgy and those of Laurence Louppe on score are taken as a starting point.

Key words: Dance, Creative writing, Dramaturgy.

\section{Un resquicio}

La danza en su forma más esencial está compuesta por movimiento y pausas. Es una manera de expresarse a través del músculo, del esqueleto, de las sensaciones totalmente subjetivas y que se escapan al otro. Una es su cuerpo y con él baila. Cierto es que nos podemos hacer una idea de lo que otros cuerpos sienten al bailar, así como podemos sentir empatía por un dolor de cabeza ajeno, un cólico, un hipo que no para, o podemos creer que compartimos lo que con toda certeza sabemos que es sentir una carcajada en el abdomen o el hormigueo de una pierna dormida. Sin embargo, la experiencia total de cada sensación es solo propia. Con esa exclusividad se danza.

Cuando aparece la palabra, escrita o hablada, hay un salto en la percepción de una misma pero también en la manera de entablar contacto con el mundo, con los otros. Aparece otra manera de relacionarse. A pesar de que las palabras pueden aludir a algo muy abstracto o metafórico, hay algo de la experiencia que se condensa en ellas. Es posible jugar con lo que se dice y con la manera de decirlo, como lo hace la literatura todo el tiempo.

En ese resquicio entre la certeza subjetiva del movimiento y la necesidad de contacto con el otro a través de un código compartido -el lenguaje- la danzaescritura encuentra su lugar. Es un espacio que permite el vaivén entre la palabra y el movimiento, entre lo individual y lo colectivo.

A partir de mi labor como bailarina y de mi interés por producir conocimiento teórico sin desvincularlo de la práctica dancística, en el 2018 abrí un primer Taller de danza y escritura ${ }^{1}$. Fue una manera de hacerle lugar a quienes estuvieran interesados en compartir los experimentos que yo había estado desarrollando durante mi investigación-creación de unipersonales los años anteriores (20132017). No se trataba de un taller de danza, sino de un espacio para que cada participante encontrara maneras de expresarse, de descubrirse y de crecer: de "auto-enunciarse". Para esto me pareció absolutamente necesaria la presencia de la escritura y la creación junto a otras personas. Estaba apostando a dos hechos que intuía: Primero, que las palabras danzando con el movimiento posibilitaban un terreno más fértil para la auto-enunciación; que la danza y la escritura eran dos actividades que hacían trabajar de distinta manera el cuerpo-mente y que la 
alternancia continua entre ellas permitía eliminar los bloqueos y dar paso a una creación más espontánea. Segundo, que la presencia de los otros era fundamental pues posibilitaba mirarnos de manera distinta a nosotros mismos; hacer visibles nuestros puntos ciegos. En ambos casos se trataba de ampliar el territorio creativo.

Una vez puestas en marcha estas dos intuiciones en la práctica de los talleres, me di a la tarea de plantear de una manera más teórica y organizada lo que sucedió en esas sesiones. En este trabajo doy cuenta de cómo se articularon el movimiento, la escritura, lo individual y lo colectivo dentro de los talleres de danza y escritura. Para describir cómo se tejen estos elementos, he tomado como concepto eje la dramaturgia, entendida esta en un sentido amplio. No solo es una herramienta para estructurar sino también un punto de partida para el análisis de la experiencia.

\section{Tejidos}

¿Cómo vincular el movimiento con la palabra, la teoría con la práctica, lo individual con lo colectivo? A través de un tejido.

La acción de tejer, como metáfora de lo que realizamos dentro de los talleres de danza y escritura, tiene que ver con entrelazar, con hacer un entramado de los distintos elementos que se ponen en juego tanto en la creación como en la elaboración teórica que dialoga con aquella. La composición de este entramado está inscrita en el marco de la dramaturgia.

Entendemos la dramaturgia como la actividad cohesionadora y reveladora de la forma y la esencia del proceso creativo y, en ese sentido, es un elemento estructurador y que propicia las auto-enunciaciones dentro del taller de danza y escritura. La dramaturgia dentro del taller está presente en diversas escalas y momentos, los cuales presentamos a continuación.

Para comenzar, tomemos la idea de la "casi nada" de la que surgen las piezas que menciona André Lepecki en su texto "No estamos listos para el dramaturgo". El autor se refiere a las obras dancísticas en las que ha participado como dramaturgo y que parten del deseo de crear pero que no tienen una ruta preestablecida: "Es en la tensión existente entre la casi-nada contenida en un deseo (llamémosle del autor, por ahora) y las realizaciones que están aún por venir (que podemos llamar obra inminente) donde opera la dramaturgia de la danza" (Lepecki, 2011, p.171). Dado que se trata de procesos con elementos heterogéneos en los que se exploran distintos aspectos (puede ser la calidad de un movimiento, una temática, un uso particular del espacio, algunas consignas creativas, un color, etc.), el dramaturgo es concebido como un "coleccionista" de los elementos que van apareciendo en el camino. Es la dramaturgia -concretada en la figura del dramaturgo-la que se encarga de hacer el tejido necesario para ir de ese "terreno confuso de dispersión" del que se parte hacia el "terreno de cohesión fragmentaria" al que se pretende llegar con la creación de la pieza. 
A lo largo del taller de danza y escritura se experimenta este tránsito de lo indefinido, de lo "nebuloso", de la "casi nada" guiada por el deseo de estar ahí y de bailar y escribir con otros, hacia las creaciones concretas hechas de movimiento y palabras. Al principio, parece que estamos dispersos y que cada uno comienza a crear desde sí mismo como si fuera un punto en ese mapa del cual todavía no se adivina el dibujo. Existe un tránsito también desde lo individual hacia lo colectivo y, una vez que se detecta esa ruta posible, los tránsitos se hacen de ida y vuelta de distintas maneras. En nuestro caso, la dramaturgia no recae en la figura de una persona sino permea el proceso y está presente en el diseño de cada sesión, en la organización de los textos y danzas individuales, en las creaciones colectivas y en el ensamblaje de las piezas para socializar la experiencia con personas externas al taller. Como las dramaturgias contemporáneas ${ }^{2}$, la nuestra está hecha en la sala de ensayo, en el foro, desde el interior del proceso mismo.

Para la ejemplificación de los aspectos en los que la dramaturgia estuvo presente durante los talleres de danza y escritura, hago referencia al taller que tomé como laboratorio propio de mi investigación. Este taller se impartió entre febrero y mayo del 2019 en el Foro Elefante, Ciudad de México, y estuvo dirigido a personas que no se dedicaran necesariamente a la danza de manera profesional. El taller se ofreció de manera gratuita y nos reunimos diez domingos entre las diez de la mañana y la una de la tarde. Si la referencia es a otro taller impartido, esto se indicará en el texto.

\section{La dramaturgia en el diseño de las sesiones}

Comencemos por la mitad. Es la sesión cinco de diez de nuestro taller en el Foro Elefante y, después de un momento de calentamiento individual, nos disponemos al trabajo colectivo. Para el calentamiento grupal los participantes toman con las manos una cuerda que se encuentra atada por los extremos. Cada uno deja caer su peso hacia atrás de modo que la cuerda esté siempre tensa. Conservando dicha tensión, cada participante propone un movimiento para calentar las articulaciones y los músculos. La tensión de la cuerda sostenida por todos es ya una insinuación metafórica del trabajo dramatúrgico a realizar durante esa y las siguientes sesiones.

Cada sesión se planea de acuerdo a ciertas actividades que se proponen a los participantes y esta planeación se hace según la experiencia de los talleres dados anteriormente pero también según el proceso que se va teniendo en el taller presente.

Las actividades son ejercicios autónomos, como si cada práctica fuera un motor independiente de los otros que echa a andar un proceso creativo. Aunque tienen esta naturaleza autónoma, los ejercicios están pensados para poder "crecer" en varias direcciones. Es por esta razón que es posible diseñar talleres de apenas unas horas y talleres que duren varios meses.

Por otra parte, cada sesión tiene un objetivo y alrededor de ese objetivo se deciden las actividades que se llevarán a cabo, procurando que haya correspondencia entre cada una de ellas. 
Regresando al ejemplo de la sesión cinco, esta fue la sesión de la mitad de nuestro proceso y en ella comenzamos a idear una estructura para, más tarde, socializar la experiencia del taller. Por esto, la dedicamos a revisar algunos de los materiales que ya se habían producido las sesiones pasadas y a ahondar en ellos con la intención de comenzar a articular estas producciones. Se trataba de un trabajo colectivo para ensamblar las individualidades y esta articulación representaba una tensión presente en el entramado que se iba construyendo.

Lepecki (2011) atribuye a la dramaturgia un papel mediador entre distintos pares de "tensiones" presentes en el trabajo creativo, algunos de los cuales ya hemos mencionado.

\begin{tabular}{|llr|}
\hline Escritura. & Vs. & Acción física. \\
\hline $\begin{array}{l}\text { El "terreno confuso de dispersión" } \\
\text { como punto de partida. }\end{array}$ & Vs. & $\begin{array}{r}\text { El terreno de "cohesión fragmentaria" } \\
\text { como punto de llegada. }\end{array}$ \\
\hline $\begin{array}{l}\text { La "casi-nada" contenida en un } \\
\text { deseo. }\end{array}$ & Vs. & La obra inminente. \\
\hline "Saber lo que la obra necesita". & Vs. & Poseer la autoría de la obra. \\
\hline
\end{tabular}

Cuadro 1. Tensiones que media la dramaturgia según André Lepecki (2011).

\section{La dramaturgia en la organización de textos y danzas individuales. Registros para la memoria y la "re-producción".}

La dramaturgia, como decíamos, se puede abordar desde distintos puntos de vista y a varios niveles. Puede provenir de alguien que testifica y guía los eventos desde "afuera" de las acciones, como en mi caso en mi lugar de coordinadora de los talleres y, también, se puede construir la dramaturgia desde "adentro", desde los que realizan las acciones; en nuestro caso, los participantes de los talleres. En el caso de los montajes escénicos, la dramaturgia puede provenir de los actores o los bailarines. Julia Varley, integrante del Odin Teatret ${ }^{3}$, afirma: "En mi trabajo como actriz, la dramaturgia es el instrumento que ayuda a organizar el comportamiento escénico, es la lógica que encadena las acciones, es la técnica para accionar de manera real en la ficción (Varley, 2009, p. 59).

Retomemos el ejemplo de la sesión cinco para hablar ahora sobre cómo los participantes organizan sus propias creaciones, las registran y vuelven a estos registros para transformarlos.

En la primera sesión del taller, cada quien creó un poema visual. Más tarde, en la sesión cinco cada participante tenía a la mano su poema. 


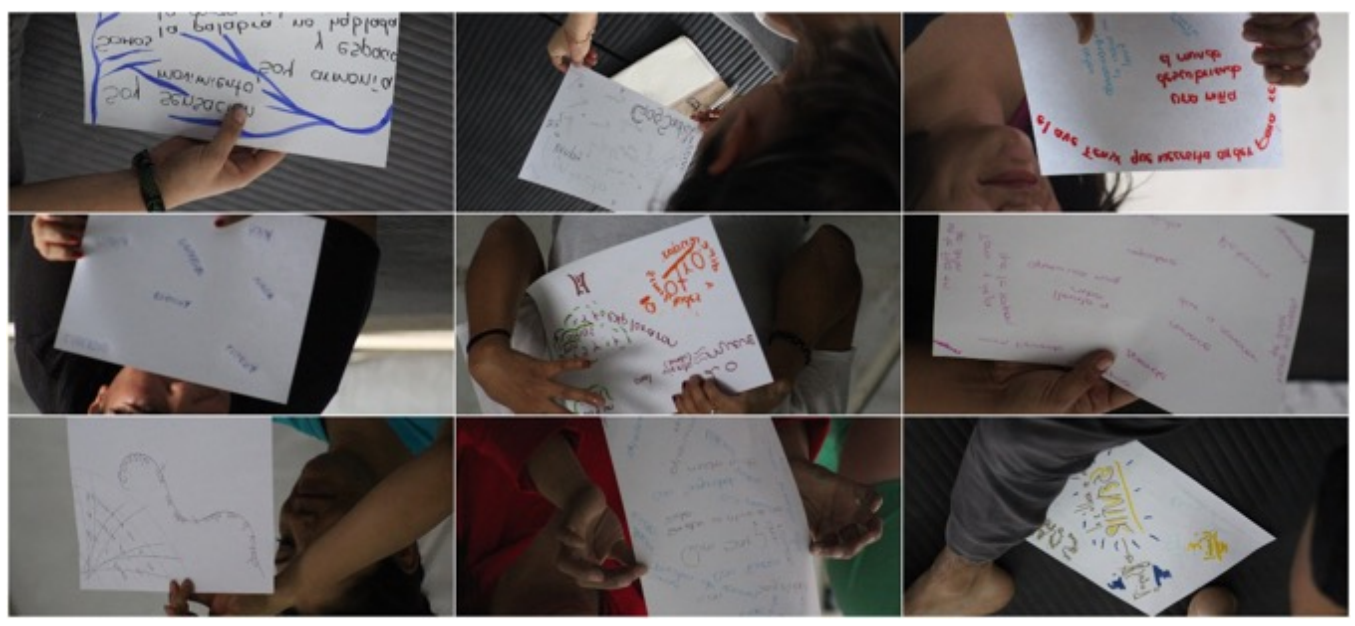

Imagen 1. Ejemplos de poemas visuales realizados en la sesión uno del Taller de danza y escritura Foro Elefante (2019). Fotografía: Abraham Rojas.

Inspirados en su propio poema visual (producción textual), los participantes crearon una danza individual con la tarea de "trasladar" eso que estaba en la hoja bidimensional al espacio tridimensional. Se aspiró a la evocación del poema y se evitó su simple ilustración o su mímica.

Las danzas creadas durante el taller no están construidas de ninguna manera por pasos o movimientos fijos; son más bien exploraciones, ensayos que al transponer un lenguaje a otro (el textual al de movimiento) revelan otro aspecto de lo creado. En este sentido, el ejercicio no consiste en hacer una "traducción" sino en tomar como punto de partida el poema visual para construir una danza que apenas lo sugiere. Podemos decir, metafóricamente, que al poema visual "le crece" una danza.

Los participantes todo el tiempo tienen una tarea creativa: trasladar el poema a su cuerpo y al espacio de la sala. En este sentido, podemos afirmar que este poema visual, de ser un producto textual, se convierte en una "partitura" en cuanto es utilizado como un "programa de actividades", aunque no explícitas ni literales.

A propósito de la partitura, la historiadora y crítica de danza Laurence Louppe (2013) ha resaltado la manera en que opera una partitura en el contexto dancístico y cómo algunos creadores han echado mano de ellas como premisas de trabajo. La partitura puede ser algo que se encuentra y se toma como tal (un ready made como lo sería una lista de supermercado o un calendario) o puede ser creada como una serie de premisas para la creación. Al respecto, Louppe menciona que "la danza establece un modelo: porque la partitura coreográfica (en mayor o menor medida aún que la partitura musical tradicional) proporciona un programa de actividades, ofrece «guiones» que a su vez generan «escenas» que se desmultiplican en el tiempo y en el espacio." (Louppe, 2013, p. 198).

El uso de partituras como premisa de trabajo da un lugar especial a la exploración personal: pone en primer plano y, al mismo tiempo, sustenta la expresión de quienes accionan. La construcción de las danzas y los textos no se realiza a través de la imitación ni la memorización sino a través de la improvisación constante y la puesta en marcha de tareas concretas, como un juego 
que tiene sus reglas y los jugadores improvisan el camino, guiados por los límites que establecen dichas reglas.

En un segundo momento, dentro de la sesión cinco, una vez terminadas las exploraciones de las danzas individuales a partir de los poemas visuales, cada participante realizó un registro de sus movimientos. El objetivo de este registro fue hacer una "nota" para la memoria. No se trataba de una descripción exhaustiva de lo que realizaron con el cuerpo sino solo de anotar algunas pistas para poder volver a su danza en el futuro. Podían valerse de la palabra o de algunos dibujos.

Laurence Louppe menciona que en un trabajo de reposición la obra no se "reproduce" en el sentido de "duplicar" sino de "producir de nuevo" (Louppe 2013, p. 198). Siguiendo esta idea, los registros que hicieron los participantes de sus danzas individuales posibilitaron que pudieran "producirlas de nuevo", aunque cada producción tenía sus propios hallazgos. En nuestro caso, la metáfora para "producir de nuevo" fue el verbo "visitar". Si se piensa la propia danza como un lugar, cada vez que se vuelve a él hay que recorrer el camino y en este recorrido es posible descubrir algo distinto cada ocasión: "La luz entra por distinto lugar a mi casa", dice Sonia, una de las participantes del taller, al referirse a lo que le sucede cuando vuelve a bailar su danza individual.

Las danzas personales creadas durante la sesión cinco fueron piezas para el juego y para el ensamblaje en las siguientes sesiones, por lo que los creadores tuvieron que repetirlas varias veces y cada vez con las mismas premisas: no buscar reproducir lo que ya se hizo sino explorar, "visitar" la danza cada vez según las nuevas condiciones.

Los participantes expresaron verbalmente cómo experimentaron la repetición de sus danzas. Es destacable que gracias a ver a los otros repetir sus propias danzas, ellos pudieron descubrir algo en las suyas:

"Ver a los demás me sirve de ejemplo para hacer más con mi cuerpo. Cinthya está ausente pero ahí está. Su cuerpo es mi cuerpo". (Tere).

"Noto las características que tiene cada quien en sus movimientos". (Manuel).

"Cada quien tiene su riqueza, su punch. Es como un rompecabezas". (Lupita I.).

“Te contagias de lo de las otras: alegría, imaginación”. (Angélica).

"Repetir sirve para mejorar, para dar intensidades diferentes. Ver los movimientos de los demás me da ideas y me inspira”. (Arlette).

"En cada danza se refleja el estado en el que llegamos a cada sesión". (Socorro).

"Es importante tener en cuenta los espacios de los que no están. Además, a veces estás perdida y de pronto te encuentras una mirada y te preguntas qué hay en esa mirada". (Lupita 0.).

"Tuve la necesidad de llevar el movimiento a otro lugar, pero no lo hice; en lugar de eso cambié el peso de mi cuerpo durante la danza". (Sonia). 
"Me cuesta trabajo tener la idea inicial de la danza”. (Fernanda).

"Me gustan las cosas no planeadas que surgen dentro de lo planeado, las coincidencias". (Maleny).

"Mi puerta me permite crear algo nuevo en el momento, dejarme ir. Lo que fue ya no es". (Erick).

“Los demás me contagian". (Carmen).

De esta manera, los textos construidos en el taller de danza y escritura tienen el potencial de ser partituras, puntos de partida para la generación de movimiento $y$, a su vez, el movimiento puede ser registrado como una serie de anotaciones personales para permitir, más tarde, volver a él.

\section{Mapas para encontrarnos en las creaciones colectivas}

Un mapa es una representación de un territorio y puede servir como guía mientras se hace el recorrido. En el caso del taller de danza y escritura, los mapas fueron creados para registrar de manera visual la procedencia —-muchas veces textual — de las danzas y el orden en el que se presentarían. De esta manera, los participantes y yo teníamos muy claro el proceso. Esta claridad permitió, de alguna manera, asir los materiales y volver a ellos de manera intencionada. En este sentido, podemos decir que los mapas que construimos funcionaron también como una suerte de partituras: "Las partituras liberan el sujeto creador porque no pretenden categorizar, ni organizar sino hacer legible el proceso. Por consiguiente, avivan la toma de conciencia artística." (Lawrence Halprin citado por Louppe, 2013, p. 199).

La toma de conciencia artística que menciona Halprin tiene que ver también con la dinámica de la creación: no se trata de un líder (dígase coreógrafo, director, coordinador, etc.) que diseña aquello que quiere ver montado y a partir de eso da instrucciones a los bailarines o performers; más bien se trata de un proceso en el que todos tienen claro el funcionamiento del dispositivo de creación y van produciendo sobre la marcha: "La partitura es el mecanismo que nos permite a todos estar implicados, hacer sentir nuestra presencia.". Las partituras son "líneas de acción en las que todos contribuyen y de las que finalmente emerge una actuación." (Louppe 2013, p. 199).

Por nuestra parte, los mapas-partitura permiten tomar cierta distancia del proceso y, por lo tanto, tener una perspectiva más clara de lo que se está haciendo. Sin duda existen muchos aspectos de la experiencia que no pueden quedar asentados gráficamente y que, quizá, ni siquiera se puedan nombrar con palabras; sin embargo, tener un referente visual de los aspectos de la creación nos ha permitido no perdernos en el camino de la producción de materiales y también ha facilitado que podamos percatarnos de cómo la creación se va "desdoblando" a partir de ciertas ideas generadoras. 


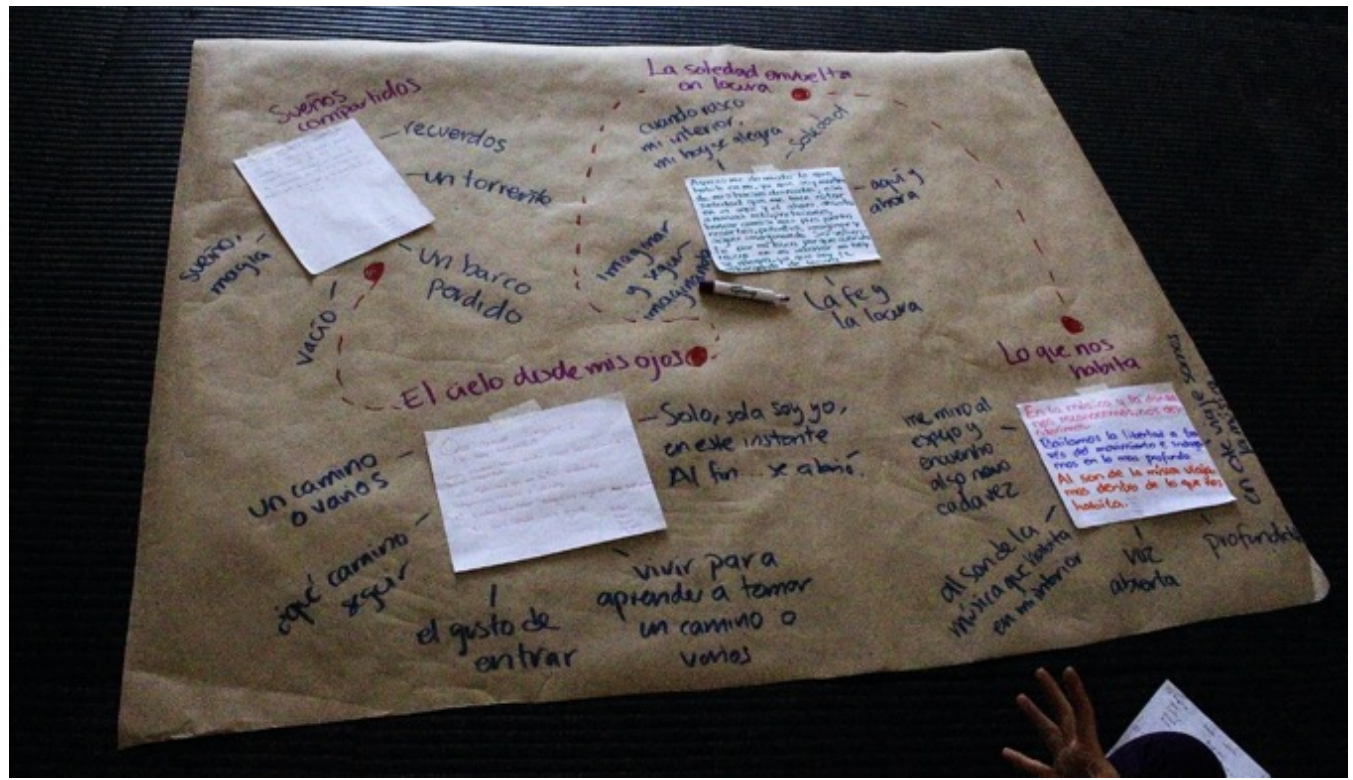

Imagen 2. Mapa creado en la sesión dos. Archivo personal.

En la Imagen 2 tenemos un ejemplo de mapa creado durante la segunda sesión. Los textos de las hojas blancas son creaciones de escritura colectiva y las frases en azul que rodean cada hoja blanca son los temas personales a partir de los cuales se harán diversos juegos creativos; a estos temas personales los llamo "puertas de entrada a la creación". Los participantes se reunieron en equipos y compartieron sus "puertas". Tras encontrar resonancias, coincidencias o divergencias entre ellas, escribieron un texto que reunía las ideas de todas y todos. Ese texto colectivo fue el punto de partida para una danza grupal. En total se crearon cuatro textos y cuatro danzas colectivas. En el mapa, en rojo y a manera de recorrido, se puede ver el orden para presentar cada danza grupal. Estas danzas grupales tienen su título en morado 4 .

Durante las sesiones cinco y seis volvimos a este mapa para tejer este fragmento con las demás producciones. Más tarde, este mapa se incorporó a otro más grande que reunía todos los materiales que presentaríamos en el Día Internacional de la Danza 2019.

Podemos decir que los mapas que creamos en el taller de danza y escritura funcionan como tales en tanto que nos ayudan a plasmar de manera gráfica el territorio que se va construyendo con las producciones textuales y de movimiento. Nos permiten distanciarnos de la creación en la que estamos inmersos y poder detectar coincidencias y resonancias en aquello que construimos, tanto a nivel individual como colectivo. Los mapas que hicimos también nos revelan la naturaleza de nuestra ruta creativa; el origen de una danza, de una palabra, de una idea y su evolución en el constante ir y venir entre el movimiento, la palabra, la exploración personal y el contacto con los otros y sus particulares maneras de crear y vivir. Nos ayudan a comprender qué hicimos y desde dónde lo hicimos.

Cuando regresamos al mapa para recordar la ruta creativa y su naturaleza, ese mapa se puede convertir en una partitura, en un "programa de acciones" que nos guía, sobre todo, cuando se trata de volver a producir. 
Sea como mapa en el momento del registro de lo que se hizo y en el momento de mirar y reflexionar sobre lo que se hizo, o sea como partitura en el momento de la repetición ("re-producción" en el sentido que explicamos más arriba), las cartografías han sido una manera muy práctica e inmediata de reconocerse en la propia creación.

\section{Ensamblar}

Cuando organicé el primer Taller de danza y escritura en febrero del 2018 no tenía en mente que dedicaríamos una sesión para mostrar nuestro trabajo a personas externas al taller. Sin embargo, sí había contemplado que la última sesión tuviéramos la oportunidad de mirar de alguna manera lo que habíamos construido; como un escultor que termina su obra y toma distancia para apreciar con otra perspectiva el trabajo realizado. Ahora me doy cuenta de que era una necesidad personal e intuitiva de sentir que de esa forma estábamos "cerrando" un proceso. Se trataba de la sensación de poder "juntar" todo eso que habíamos hecho y hacer un recuento práctico de lo que habíamos experimentado. Así que pensaba la última sesión (en aquella ocasión solo fueron cuatro) como una presentación organizada de nuestras creaciones para nosotras mismas.

De manera espontánea, en algún momento de la tercera sesión, las participantes expresaron el deseo de tener algunos invitados cercanos para esa última reunión, así que al final cada una fue acompañada por una o dos personas y les mostramos tres danzas colectivas que unían los textos y las danzas individuales. En las paredes de la sala colgamos nuestros apuntes, nuestros mapas, dibujos, "puertas" y textos. Incluso, un equipo incorporó a su danza un pliego de papel que contenía dibujos de la silueta de partes de su cuerpo y algunas frases.

En el segundo taller, que también fue de cuatro sesiones (septiembre-octubre 2018) no surgió la inquietud de invitar a alguien y más bien hicimos un convivio al final para charlar sobre nuestras experiencias.

Con estos antecedentes, para el taller que se llevó a cabo en el Foro Elefante (febrero-mayo 2019), y que, insisto, sirvió como laboratorio para esta investigación, desde el principio consideré mostrar el trabajo, aunque pensé que sería una decisión que tomaríamos en grupo una vez comenzado el curso. La presentación no la concebía como el objetivo del taller sino como otro experimento dentro del mismo. Se trataba de compartir con otros las producciones en un ambiente de confianza y convivio. Además, retomar lo que habíamos hecho y organizarlo, también permitía tener cierta perspectiva y distancia, lo cual podría contribuir a la toma de consciencia sobre ciertos aspectos de la propia creación y contribuir al autoconocimiento.

Una vez decidido que sí socializaríamos nuestro trabajo, hice los siguientes planteamientos: 


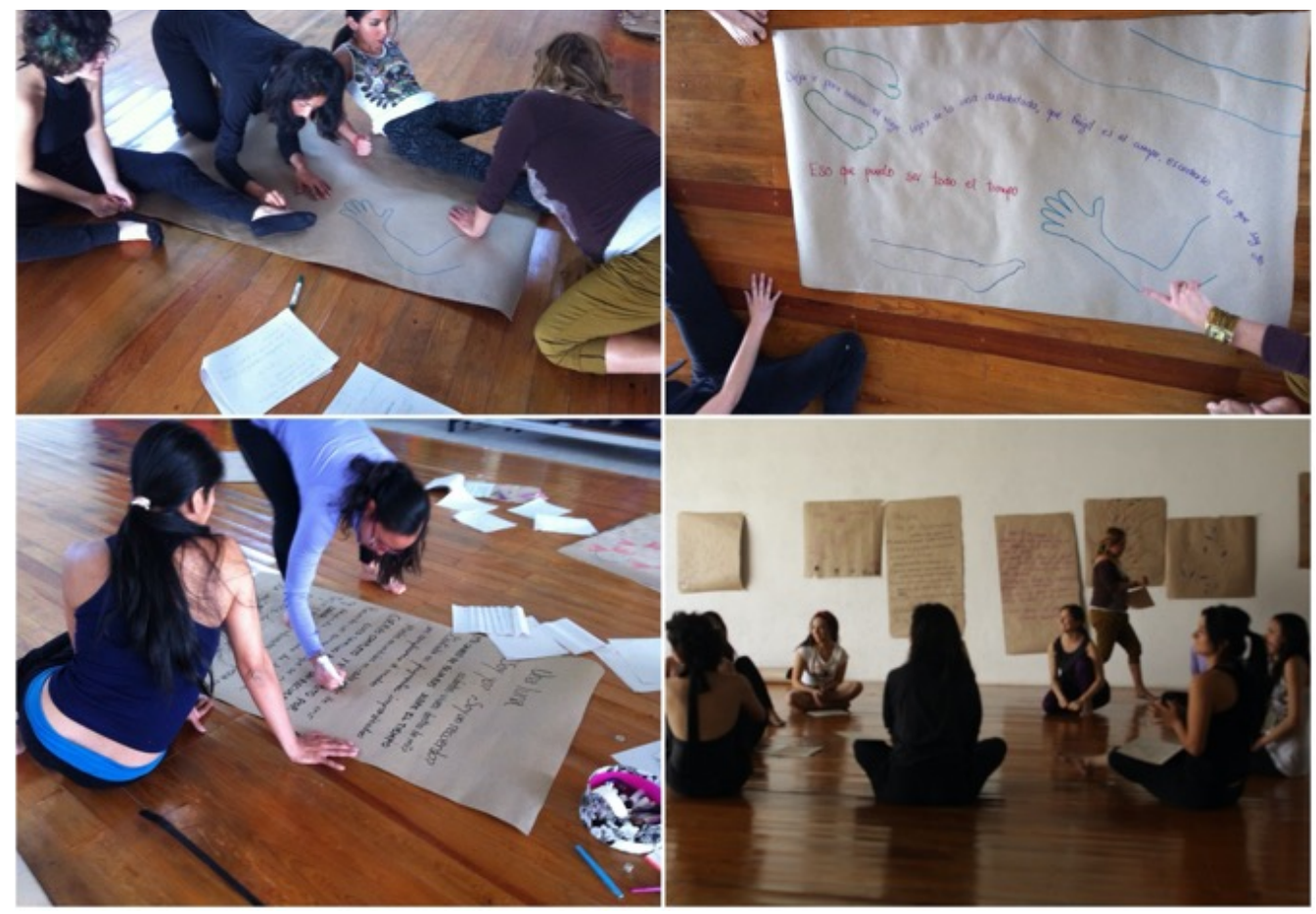

Imagen 3: Primer Taller de danza y escritura. Ciudad de México, febrero 2018. Archivo personal.

1. Algunas sesiones las dedicaríamos a la producción y exploración de materiales a través de ejercicios de escritura y movimiento (sesiones de la uno a la cuatro).

2. Otras sesiones estarían enfocadas en la reelaboración y organización de los materiales producidos (sesiones de la cinco a la ocho).

3. La presentación la haríamos en el marco del Día Internacional de la Danza 2019 en el Foro Elefante (sesión nueve).

4. La última sesión estaría dedicada a hacer un cierre de todas las experiencias del taller.

De acuerdo a esto, la dramaturgia estuvo presente en el ensamblaje de los materiales para el día de la presentación. No se trató de planear una secuencia de cuadros con los materiales producidos, sino que siguió la misma lógica y principios que las primeras sesiones: nos adentramos a esta parte del proceso con un ánimo de experimentación, de disposición lúdica. Tomamos los materiales ya producidos, los intervenimos y los hicimos dialogar con otros materiales, ya fueran danzas (bailadas en vivo o grabadas) o textos.

Como coordinadora del taller me enfrenté a la cuestión sobre cómo hacer ese "armado" de los materiales, cómo ensamblarlos, cómo hacerlos convivir sin que fuera caótico, pero que tampoco cayera en lo mecánico y obvio.

André Lepecki también se preguntaba cómo se ensambla y encontró un atisbo de respuesta en el fragmento de un texto de José Gil: 
El deseo crea ensamblajes. Pero el movimiento hacia esos enlaces siempre abre camino a nuevos ensambles. Y esto es porque el deseo no se agota en su misma satisfacción como crece ensamblándose de nuevo. Y todo, para crear nuevas conexiones entre materiales heterogéneos; establecer nuevos vínculos, otras vías para canalizar la energía; conectar, poner en relación, hacer simbiosis, provocar que algo ocurra, crear máquinas, mecanismos, articulaciones; esto es lo que significa ensamblar. Exigir constante e incesantemente nuevas relaciones. (José Gil en Lepecki 2011, p.174)

Establecer nuevas relaciones entre materiales heterogéneos: ¿No es esa la esencia de la metáfora? En cada taller que he dado he comenzado la primera sesión hablando de la metáfora y aludo a su etimología, "trasladar más allá", para invitar a los participantes a moverse cada vez que sea posible hacia el terreno de lo metafórico, porque es en esa tierra no agotada que se pueden crear relaciones entre ideas, movimientos, palabras y seres humanos. Estas relaciones son las que nos revelan una parte del mundo y de nosotros mismos que hasta ese entonces ignorábamos. Es en ese proceso creativo de encontrar la resonancia de las palabras propias en las del otro, de la danza de un cuerpo en los cuerpos ahí congregados que puede tener cabida una enunciación que cobra sentido tanto para quien la realiza como para quien la recibe. Es también en esa labor de ensamblaje, de poner en relación diversos materiales, que entre el "yo" y el "tú" se comienza a fraguar un "nosotros".

Habría que esclarecer a qué se refiere el deseo como principio de ensamblaje. En mi caso, y como yo lo concibo, el deseo está en la voluntad de cada participante para estar en el taller, para vincularse con otros y para mostrar algo de sí mismos que pueda ser detonante para la creación colectiva. Como mediadora del taller, buena parte de mi labor reside en crear el ambiente adecuado de confianza para mantener vivas esas voluntades.

En las sesiones que dedicamos a ensamblar los materiales para la presentación, llevamos a cabo algunos experimentos. Como decía, no se trató de acomodar un material después de otro, sino de "visitar" los materiales que teníamos y ponerlos en diálogo con algún otro elemento. Por ejemplo, durante la sesión cuatro creamos cuatro videos (uno por grupo) ${ }^{5}$ a partir de las "puertas" ajenas ${ }^{6}$ y durante la sesión siete hicimos unas improvisaciones con los videos proyectados en la pared como interlocutores del movimiento en vivo. Se trataba de componer en tiempo real a partir de las frases de las "puertas" con las que se crearon los videos. Este momento de improvisación fue incluido en la estructura final para la presentación.

De esta manera, la necesidad de ensamblar los materiales surgió como recurso para compartir la experiencia del taller con algunos invitados. La dramaturgia, en este caso, funcionó según lo que menciona Lepecki (2011): para dar consistencia, solidez y coherencia estética a la pieza; en nuestro caso, a "Yo, tú, nosotros", que fue como nombramos la obra que presentamos ante el público. 


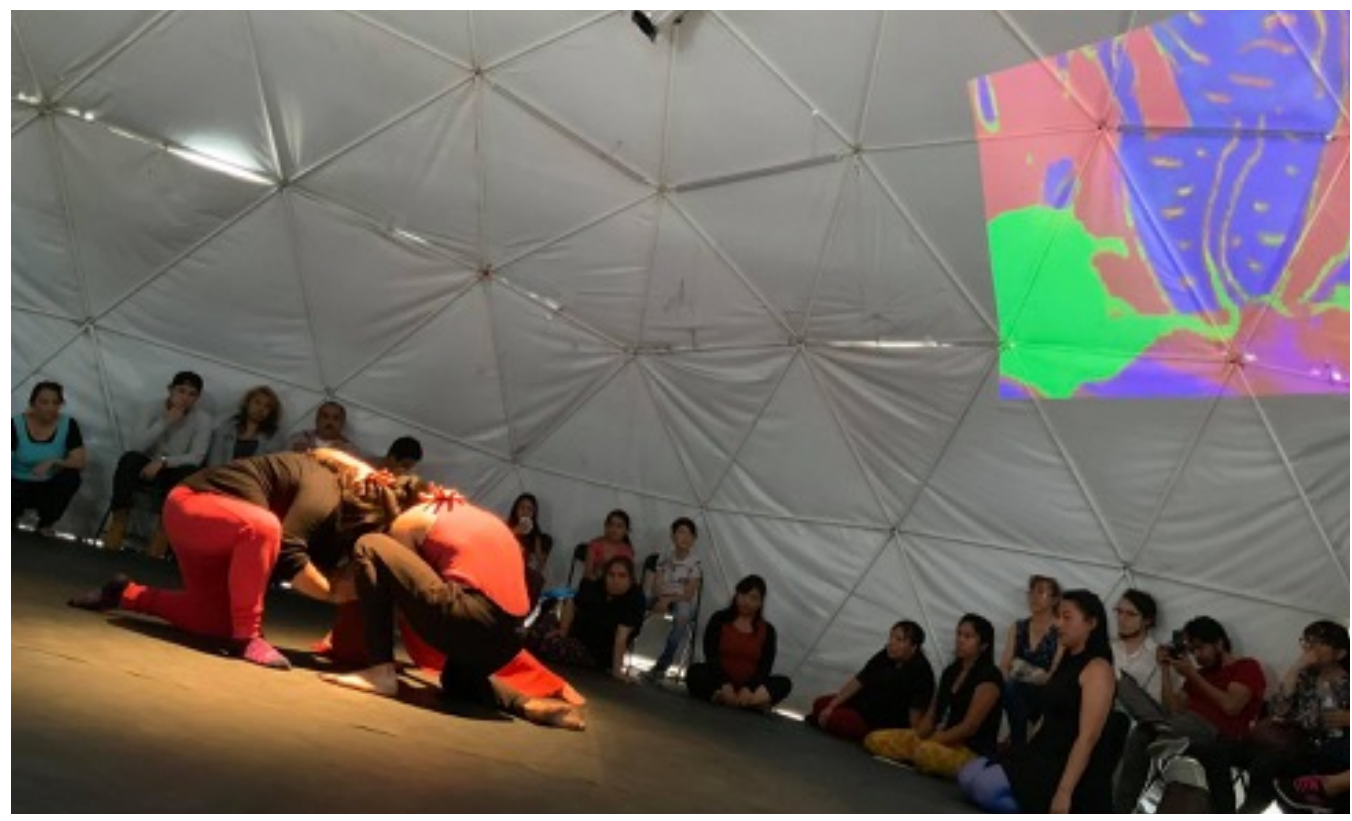

Presentación de la pieza "Yo, tú, nosotros" en el marco del Día Internacional de la Danza 2019. Foro Elefante, CDMX. Fotografía: Abraham Rojas.

\section{Conclusiones}

La dramaturgia como concepto articulador y como herramienta práctica la encuentro en el diseño de las sesiones del taller de danza y escritura; en el acompañamiento que hice de los procesos creativos tanto individuales como colectivos; en las dramaturgias que los participantes hicieron en sus creaciones (también tanto individuales como colectivas); en la realización de una estructura para compartir el proceso y presentarlo ante más personas; en el tejido entre escritura y movimiento y, finalmente, como instrumento para ordenar la autoenunciación.

La intención de los talleres de danza y escritura desde su inicio fue la de propiciar un espacio creativo para que las personas, sobre todo las que no se dedicaran profesionalmente al campo dancístico, pudieran experimentar el movimiento y la escritura como un medio para su auto-enunciación y que este acto tuviera un impacto positivo en su experiencia vital.

La apertura de esos espacios de creación estuvo guiada en todo momento por mi intuición y mis experiencias previas. Una vez que llevé estas experiencias al campo de la investigación académica, me ha resultado sorprendente la revelación del complejo mapa de conexiones entre práctica y teoría que se derivan de la realización de los talleres. Lepecki (2011) defiende la postura del dramaturgo como alguien que se aproxima a la obra desde un "no saber" y con un constante "errar" como naturaleza de esta aproximación. Por mi parte, considero que la investigación que llevo a cabo implica también una zona amplia de "no saber" de qué va exactamente el trabajo hasta que, con el tiempo y la elaboración constante, va apareciendo su forma. Este texto ha ayudado a vislumbrar una parte del dibujo de mi investigación y a entablar el diálogo entre la producción teórica y la experiencia práctica. 
${ }^{1}$ Desde el 2018 a la fecha (mayo 2021) he dado cinco talleres de danza y escritura. El más corto duró cuatro horas repartidas en dos sesiones (Universidad de Lille, Francia, noviembre 2019) y el más largo duró diez sesiones de tres horas cada una con un encuentro por semana (Ciudad de México, febrero-mayo 2019). Este último sirvió de laboratorio para el desarrollo de mi investigación doctoral "Investigación-acción: La danza-escritura como posibilidad de auto-enunciación".

${ }^{2}$ Para más información sobre las dramaturgias contemporáneas se puede consultar la compilación de textos Repensar la dramaturgia. Errancia y transformación. España: Centro Párraga, 2011.

${ }^{3}$ Julia Varley forma parte del Odin Teatret desde 1976. Esta agrupación teatral dirigida y fundada por Eugenio Barba tiene actividad desde 1964 y ha logrado mantener a sus miembros durante varios años (en algunos casos, décadas), lo que ha propiciado que sus actores tengan un trabajo de investigación e interpretación bastante sólido. Aunque Varley continúa colaborando con la compañía, tiene su propio proyecto profesional que incluye, además de la creación, la docencia y la escritura.

${ }^{4}$ Es posible ver las danzas producidas y más material sobre el proceso de los talleres en el blog del proyecto: https:// dansescritura.blogspot.com/

${ }^{5}$ Estos videos también están disponibles en el blog del proyecto: https://dansescritura.blogspot.com/2019/04/ videodanzas-videodanses.html

${ }^{6}$ Recordemos que llamamos "puertas de entrada a la creación" a los temas individuales de los que cada participante partió para varios ejercicios.

\section{Referencias bibliográficas}

Lepecki, A. (2011). No estamos listos para el dramaturgo: Algunas notas sobre la dramaturgia de la danza. En Repensar la dramaturgia. Errancia y transformación (pp. 161- 179). España: Centro Párraga. Disponible en:

https://labencrisis.files.wordpress.com/2013/07/repensar-texto-justificado-1-1.pdf

Louppe, L. (2013). Partituras. En Lecturas sobre danza y coreografía (pp. 197-208). Madrid: Artea Editorial.

Varley, J. (2009). Piedras de agua. México: Instituto Queretano de la Cultura y las Artes/ Escenología. 